\title{
LA LEY COMO ACCIÓN DE LA AUTOCONCIENCIA MORAL EN LA ÉTICA DE HERMANN COHEN
}

\author{
Héctor Óscar Arrese Igor \\ Universidad Nacional de La Plata (Argentina)
}

RESUMEN. La ética de H. COHEN ha recibido la crítica de ser un mero formalismo abstracto, lo que implica una desconexión entre el individuo particular y la universalidad de la ley. En este trabajo intento mostrar que la filosofía moral de COHEN resiste estas críticas, porque el concepto de ley particular integra a la individualidad de cada ciudadano con la universalidad de la autoconciencia, que se expresa en el Estado.

Palabras clave: H. COHEN, formalismo abstracto, universalidad de la ley, filosofía moral.

ABSTRACT. H. COHEN's ethics has received the critique of being a mere abstract formalism, wich implies a disconnection between the particular individual and the universality of the law. In this paper I try to show that COHEN's moral philosophy can resist these critiques, because the concept of particular law integrates the individuality of each citizen with the universality of self-conciousness, wich expresses itself in the state.

Keywords: H. COHEN, abstract formalism, universality of the law, moral philosophy.

\footnotetext{
* Fecha de recepción: 24 de febrero de 2010. Fecha de aceptación: 28 de marzo de 2010
} 


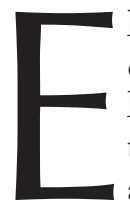

I neokantismo decimonónico ha florecido, entre otros lugares, en la Universidad de Marburg, bajo el profesorado de H. COHEN y de discípulos suyos de la talla de P. NATORP. COHEN intentó dar respuesta a los desafíos que planteaba la cultura de aquel momento, en particular al desarrollo notable que adquirieron las ciencias naturales y sociales ${ }^{1}$. Contra el psicologismo de su maestro F. A. LANGE, COHEN consideró que las categorías fundamentales de la filosofía no pueden ser reducidas a procesos biológicos del cerebro, sino que el pensamiento filosófico tiene una función trascendental, que consiste en la fundamentación de los conceptos fundamentales que están a la base del corpus científico ${ }^{2}$. En esto consiste su propia interpretación de la filosofía de KANT, desarrollada ya en sus primeras obras: Kants Theorie der Erfahrung (La teoría de la experiencia de Kant, 1871), y Kants Begründung der Etbik (La fundamentación de la ética de Kant, 1877). De acuerdo con estos textos, el método trascendental consta de dos momentos: en primer lugar, se parte de un conjunto de conceptos sistematizados en un corpus científico y, luego, se lo intenta explicar en base a determinadas categorías fundantes, entendidas como su condición de posibilidad.

La ciencia, desde el análisis de COHEN, tiene una estructura legaliforme, cuyas últimas categorías consisten en normas epistémicas que dan razón de las leyes que la componen. Ahora bien, COHEN no construyó un sistema filosófico monista donde no hubiera lugar para la diversidad del conocimiento científico. Por el contrario, su sistema filosófico está dividido claramente en dos partes: una dedicada a la filosofía teórica (encargada a su vez de fundamentar a las ciencias matemático-naturales) y otra consagrada a la filosofía práctica (cuyo objeto son las ciencias sociales, que se fundan en la ciencia del Derecho).

La ética de COHEN intenta dar cuenta entonces de las condiciones de posibilidad de la legalidad propia de la ciencia del Derecho. El sistema del Derecho, sostiene CoHEN, es idéntico al Estado, porque este último no es otra cosa que el conjunto de normas que estructuran la vida de la comunidad. Es decir, que el Estado contiene en su propia Constitución tanto las normas para producir la ley (que prescriben cómo debe funcionar el poder legislativo, etc.), cuanto las que son necesarias para su aplicación (asunto del poder ejecutivo y judicial). Esto es posible gracias a una doble dimensión que recorre todo el Estado porque, por un lado, el Derecho tiene una forma contractual y, por el otro, tiene como contenido a una determinada forma de subjetividad.

Veamos cada dimensión por separado. La forma contractual del Derecho viene dada por las condiciones de su producción. Es decir, que la actividad de la legislación en el Parlamento supone el acuerdo libre entre adultos consintientes en condiciones de simetría. Dicho de otro modo, la legislación no tiene legitimidad en un Estado democrático de Derecho si no está a cargo de los representantes del pueblo, elegidos por medio del sufragio universal. Pero el Derecho no sólo tiene una forma, sino que también tiene un contenido, que consiste en la expresión de la voluntad del Estado, como producto de la actividad de la legislación. El problema que debe enfrentar COHEN al

\footnotetext{
Al respecto, cfr. KÖHNKE, 1986; SIEG, 1994, y WILLEY, 1978.
}

2 Cohen, 1914. 
determinar el contenido de la voluntad del Estado consiste en establecer el status del sujeto de esta voluntad, que es el Estado mismo.

En primer lugar, COHEN entiende al Estado como una persona jurídica, esto es, como una persona con una voluntad puramente ficticia y jurídica, que consiste en una serie de normas que la regulan, pero también en una serie de reglas acerca de cómo deben elaborarse y aplicarse estas normas. La voluntad del Estado no se funda entonces en ninguna cosmovisión religiosa ni en ningún conjunto de tradiciones políticoculturales que pudieran entenderse como el «espíritu del pueblo» (Volksgeist). Por el contrario, el Estado se funda en esta subjetividad universal que constituye su personalidad jurídica, denominada por COHEN como «autoconciencia» (Selbstbewusstsein) ${ }^{3}$. La autoconciencia pone en relación a cada individuo con la universalidad de la humanidad, en la persona de sus conciudadanos. Se trata de una comunidad espiritual, que unifica a todos los ciudadanos por los lazos de la ley que ellos mismos han producido en la persona de sus representantes y a la que ellos también se comprometen a someterse $^{4}$.

COHEN estructura el concepto de la autoconciencia en base a la tarea, en tanto que es el motivo de la voluntad pura. Como dice COHEN,

«El concepto de ley, al que aquí se está haciendo referencia, no tiene que ver con la unidad y la esencia de las leyes, que son establecidas por la ley fundante, la Constitución; sino más bien con las leyes particulares, en las cuales se atestiguan y se realizan las tareas de la voluntad estatal. Sin ley no hay voluntad, y por tanto tampoco una autoconciencia del Estado. Este concepto estatal de la ley debe convertirse en el concepto guía para la autoconciencia personal» ${ }^{5}$.

La tarea de la autoconciencia en la voluntad estatal es el objetivo último de la moralidad que, a su vez, es el norte de la vida política. Nunca puede decirse que se ha completado la tarea de la autoconciencia, porque es infinita y está siempre abierta al futuro ${ }^{6}$. Dicho de otro modo, la autoconciencia es el sujeto de la voluntad pura sólo en la medida en que actúa ${ }^{7}$. Si bien COHEN sostiene una ética universalista de inspiración kantiana, lucha a lo largo de su Ethik des reinen Willens para superar el mero formalismo que significaría la postulación de una legalidad abstracta sin contacto alguno con la individualidad concreta de cada ciudadano.

La instancia superadora del formalismo en la teoría de COHEN es el concepto de autonomía moral. Se trata de las condiciones que deben darse para que el individuo pueda participar en la tarea de la autoconciencia, que van desde la autolegislación,

$3 \operatorname{ErW}, 201-257$.

$4 \operatorname{ErW}, 357-372$.

5 «Bei dem Gesetze, wie es hier gemeint ist, handelt es sich aber nicht um die Einheit und den Inbegriff der Gesetze, den das Grundgesetz, die Verfassung ausmacht; sondern um die einzelnen Gesetze selber, in denen die Aufgaben des Staatswillens sich bekunden und betätigen. Ohne Gesetz kein Wille, also auch kein Selbstbewusstsein des Staates. Dieser Staatsbegriff des Gesetzes muss der Leitbegriff werden für das persönliche Selbstbewusstsein» $(E r W, 262)$.

6 Para el Estado como Zielbegriff de la autoconciencia, cfr. SCHMID, 1993: 72-73; LISSER, 1922: 56-57.

7 «El producto y el testimonio de la voluntad pura es la acción. A ella corresponde y de ella surge la autoconciencia. La acción representa mejor y con más claridad la producción de la autoconciencia» (《Das Erzeugnis und das Zeugnis des reinen Willens ist die Handlung. Ihr entspricht, ihr entspringt das Selbstbewusstsein. Mehr und deutlicher als der Wille stellt die Handlung die Erzeugung des Selbstbewusstseins dar»; $E r W, 259)$. 
pasando por la autodeterminación y la autorresponsabilidad, hasta llegar finalmente a la autoconservación ${ }^{8}$. La última condición de posibilidad de la autoconciencia es entonces que el ciudadano pueda autoconservarse en la existencia, es decir, que esté en condiciones de satisfacer sus necesidades básicas. Pero entre la personalidad universal de la autoconciencia y la particularidad del individuo singular se ubica el concepto de ley particular, que exige a su vez la autonomía moral de aquellos ciudadanos que produzcan la ley y luego se sometan a ella.

Algunos intérpretes, como J. KLEIN o E. WINTER, han objetado que la ética de COHEN no lograría integrar satisfactoriamente los planos de la individualidad y la universalidad $^{9}$. No se trata de una objeción menor porque, si ambos planos están desconectados, entonces la teoría no podrá aplicarse y perderá todo sentido en tanto que filosofía moral. En este trabajo intentaré determinar el concepto de ley que COHEN sostiene y, de este modo, mostrar cómo se da la interrelación entre el individuo y la universalidad en la ErW.

\section{LA LEY COMO LA ACCIÓN DE LA AUTOCONCIENCIA}

COHEN emprende el análisis del concepto de la acción con el propósito de liberar definitivamente a la autoconciencia del prejuicio psicologista y, de este modo, desvincularla de toda fundamentación en el yo empírico. La autoconciencia puede ser demostrada de manera adecuada sólo una vez que se ha establecido su realización por medio de las leyes particulares en el Estado ${ }^{10}$. Sin embargo, puede surgir un conflicto entre las convicciones morales del individuo y el contenido de la ley. Es decir, que puede aparecer una tensión entre la particularidad del ciudadano y la universalidad de la ley. Por ejemplo, podría impugnarse cierta ley particular, por considerarla injusta en relación con otras leyes naturales o leyes no-escritas, que operarían de este modo como criterio moral frente al Derecho positivo.

Sin embargo, CoHen piensa que no puede justificarse la moralidad o la inmoralidad de las leyes positivas en base a una legalidad natural previa al Estado, sino que deben serlo simplemente en virtud de las disposiciones mismas establecidas en la Constitución, que marcan cómo debe legislarse y aplicarse la ley para constituir al sujeto colectivo de la autoconciencia. La autoconciencia es entonces el concepto guía para juzgar la moralidad de las leyes del Estado, por lo que se trata de un criterio inmamente al sistema legal mismo.

Es en este contexto que COHEN remite al ejemplo histórico de Sócrates, quien reconoció la majestad del Estado en la ley injusta que lo condenaba a muerte ${ }^{11}$. Sócrates prefirió la vigencia de una ley injusta a la ausencia total de leyes y a la destrucción del Estado. Por eso no juzgó a sus jueces de acuerdo con leyes naturales, independientes de la legislación estatal, sino que más bien decidió aceptar su veredicto y se rehusó a seguir la sugerencia de Critón de sobornar a los guardias, a fin de escapar y marchar

\footnotetext{
$8 \mathrm{ErW}, 324-388$.

9 KLEIN, 1976: 17; WINTER, 1980: 394-425.

10 WINTER, 1980: 349 .

${ }^{11}$ Cfr. SCHMID, 1993: 71-72.
} 
al destierro ${ }^{12}$. Sócrates comprendió, entonces, que es preferible una vida injusta como ciudadano de un Estado democrático que una vida por fuera de la comunidad estatal.

La razón de esto es que Sócrates entendió que la participación en la comunidad espiritual de la autoconciencia es la condición necesaria para llevar una vida moral. Es decir, que el ciudadano puede superar el punto de vista egoísta del sujeto aislado, y ponerse en correlación con la universalidad de los demás, sólo cuando acepta y cumple la ley dictada por el Estado ${ }^{13}$. La ética —que tiene por objeto la correlación entre el individuo y la universalidad - sólo puede ofrecer a cada ciudadano el concepto guía de la autoconciencia, que se realiza en el Estado ${ }^{14}$.

Como es evidente, a partir de su análisis del concepto de autoconciencia, COHEN concluye que existe una identificación íntima entre la ética y el sistema legal. Mejor dicho, COHEN sostiene que la ética provee al sistema legal de las categorías fundamentales para explicar su constitución última, lo que vale tanto para los conceptos de voluntad pura y autoconciencia, como para el concepto de acción ${ }^{15}$. Por eso rechaza la división tajante de KANT entre ética y Derecho ${ }^{16}$. COHEN considera que la filosofía moral defendida por KANT no puede unificar a la ética con el Derecho, porque no dispone de un factum científico como punto de partida ${ }^{17}$.

En el análisis de COHEN, la filosofía del Derecho de KANT, a diferencia de su ética, tiene un fundamento externo de determinación de la voluntad. Pero COHEN considera que la reclusión de la ética en el foro interno de la conciencia, y su consiguiente fundamentación en la convicción (Gesinnung), abren el camino para el imperio de la religión en un ámbito de la conciencia cultural, que en realidad debería confiarse solamente al conocimiento científico del Derecho ${ }^{18}$.

A fin de colocar sobre fundamentos firmes el vínculo íntimo que reclama para la ética y para el Derecho, COHEN investiga el rol que juega la coacción en la realización de la autoconciencia en el Estado. Si consigue mostrar la relevancia moral de la coacción legal, podrá superar entonces la división kantiana entre la ética y el Derecho, y demostrar el carácter trascendental de la ética respecto del sistema jurídico. Veamos si cumple con su cometido.

COHEN sostiene que la función que cumple la coacción, desde el punto de vista legal, no imponer arbitrariamente la autoridad del Estado, sino más bien garantizar la conciliación de las voluntades o los arbitrios del yo y del tú ${ }^{19}$. Es decir, el Derecho está

${ }^{12}$ ErWW, 264-265. Para la recepción del pensamiento de SÓCRATES y PLATÓN en COHEN, cfr. LEMBECK, 1994.

13 Éste es uno de los sentidos que COHEN le da al concepto de la autonomía moral como autorresponsabilidad (ErW, 357-372).

${ }^{14}$ Cfr. el análisis de la autoconciencia como concepto guía del Estado en SCHMID, 1993: 72-73; LISSER, 1922: 56-57.

${ }^{15}$ Con palabras de COHEN: «La ética debe realizarse a sí misma como una filosofía del Derecho» («Die Ethik muss selbst als Rechtsphilosophie durchführen», ErW, 225).

${ }^{16} C f r$. WINTER, 1980, 253. Obvio es decir que esta particular interpretación de COHEN de la relación entre ética y Derecho en KANT podría ser refutada, y de hecho lo ha sido. Aunque no es mi objetivo entrar en cuestiones interpretativas de la filosofía práctica kantiana.

17 Guariglia, 1992: 58.

$18 \mathrm{ErW}, 269$.

19 «¿Qué significado puede tener la coacción para el Derecho? Para dar cuenta de esta reflexión no se toma en cuenta el éxito de la coacción; este éxito no es algo esperado. Se trata exclusivamente de la característica que de este modo debe recibir el concepto del Derecho, para unificar la arbitrariedad de uno con la del 
allí para llevar a buen término los conflictos entre los ciudadanos, en torno a determinados privilegios, recursos, etcétera ${ }^{20}$. Sin la coacción, la ley que rige el Derecho no puede ser aplicable y el Estado pierde toda realidad histórica, porque deja de ser una persona jurídica.

Pero el problema de la ética no es otro que el de la conciliación de la libertad del yo y del tú según leyes universales ${ }^{21}$. Por esta razón, COHEN sostiene que el carácter coactivo del Derecho, en vez de separar al Derecho de la ética, los une íntimamente. La coacción no es otra cosa que la aplicación consecuente de la ley, que ha sido promulgada por los mismos sujetos a los que es aplicada ${ }^{22}$. Por eso COHEN rechaza la teoría de los imperativos de E. N. BIERLING, según la cual la ley tiene la forma de una prescripción impuesta por el legislador al pueblo, que podría formularse como la oración «yo quiero, tú debes» ${ }^{23}$. En realidad la autoconciencia del Estado nos señala más bien que la legislación no es el producto de un grupo social particular, sino del «nosotros» que unifica al yo y al tú en una misma voluntad ${ }^{24}$. Dicho de otro modo:

«Y puede hacerse llegar a cada uno el llamado: tú debes; y no se debe para ello tomar por separado al yo del legislador. En el reconocimiento exigido se manifiesta una atribución para la prescripción. En la tarea de su autoconciencia, podremos decir, yace el fundamento legal de la norma» ${ }^{25}$.

Las teorías de COHEN y de BIERLING coinciden en que las normas legales tienen la forma de un imperativo o de una prescripción. Por esta razón se apartan de la teoría de E. Zitelmann, quien considera que las normas legales son en realidad juicios descriptivos sobre el funcionamiento de las instituciones ${ }^{26}$. Es decir, ZiTELMANN entiende a las normas legales no ya como enunciados prescriptivos, sino más bien de tipo indicativo. Sin embargo, objeta COHEN, las normas legales exigen el cumplimiento de determinadas conductas, que son los efectos legales que se espera que ocurran ${ }^{27}$.

otro. Se trata fundamentalmente también de mediar en el conflicto entre el uno y el otro» («Welche Bedeutung kann nun aber der Zwang für das Recht haben? Auf den Erfolg des Zwanges, um diesen Gedanken zunächst abzufertigen, ist es keineswegs abgesehen; der wird nicht erwartet. Es handelt sich lediglich um das Merkmal, welches der Begriff des Rechtes dadurch empfangen soll, um die Willkür des Einen mit der Willkür des Andern zu vereinigen. Es handelt sich also im Grunde nur um den Gegensatz des Einen und des Andern, der geschlichtet werden soll», ErW, 269).

20 WINTER, 1980, 255.

21 Aquí resuena el eco de la definición kantiana del Derecho, en la Metafísica de las Costumbres, que reza: «Una acción es conforme a Derecho (recht) cuando permite, o cuya máxima permite a la libertad del arbitrio de cada uno coexistir con la libertad de todos según una ley universal» («Eine jede Handlung ist recht, die oder nach deren Maxime die Freiheit der Willkür eines jeden mit jedermanns Freiheit nach einem allgemeinen Gesetze zusammen bestehen kann») (AA, VI, 230). Cito por la trad. esp. de Adela Cortina Orts y Jesús Conill Sancho, en KANT, 1997: 39.

22 Este punto de la teoría de COHEN tiene notables semejanzas con la teoría pura del Derecho de KANT, como puede constatarse leyendo MdS, AA, VI, 335.

${ }^{23}$ BIERLING, 1894 y ss.

${ }^{24}$ Cfr. WINTER, 1980: 365.

25 «Dann kann aber auch jedermann an sich selbst den ruf ergehen lassen: du sollst; und er braucht dazu nicht erst das Ich des Gesetzgebers hinzuzunehmen. In seiner Anerkennung, die erfordert wird, gibt sich eine Befugnis zur Verordnung kund. In der Aufgabe des Selbstbewusstseins, werden wir sagen dürfen, liegt der Rechtsgrund der Norm» (ErW, 271).

${ }_{26}$ Puede verse al respecto Zitelmann, 1879.

27 WINTER, 1980: 369. 
Es decir que, dado que las normas legales son prescripciones que reclaman un cumplimiento perentorio, están orientadas a la producción de determinadas consecuencias ${ }^{28}$. Las leyes de la ciencia natural, en cambio, no acarrean otras acciones en términos de efectos, sino que más bien se limitan a explicar y describir cómo de ciertos fenómenos se siguen otros de acuerdo con regularidades ${ }^{29}$. ZitELMANN funda su teoría en un elemento común a las normas legales y las leyes naturales, que induce a una identificación de ambos tipos de legalidad. Se trata del concepto de la condición. El problema radica en que los dos tipos de leyes aparentemente no podrían distinguirse, porque ambos contienen en sí las condiciones de su aplicación.

COHEN sostiene que las leyes naturales están formuladas correctamente, cuando es posible identificar cada caso particular que cae bajo cada ley. Sólo de este modo puede llevarse a cabo una explicación nomológico-deductiva, porque esta tarea consiste en la derivación del caso particular a partir de una ley y de ciertas condiciones iniciales. La norma legal también contiene en sí las condiciones que deben cumplir los casos particulares para quedar tipificados en la ley particular y, por tanto, garantizar su aplicación. Sin embargo, hay una diferencia substancial entre las dos formas de razonamiento condicional.

Las normas legales se fundan sobre una serie de condiciones, que son las exigencias estipuladas por la Constitución para regular la actividad de la legislación. Asimismo, las normas legales también establecen una serie de reglas para sancionar las transgresiones a la ley que están tipificadas en ellas. Las condiciones legales prescriben entonces la ejecución de ciertas acciones, tanto en lo relativo al cumplimiento cuanto a la transgresión de la ley.

La categoría de la condición confiere a las leyes del Derecho su universalidad, esto es, su aplicabilidad a todos los casos que tipifican, sin excepciones. La universalidad de la ley no resulta de su mera forma, con abstracción de su materia, sino que más bien se trata de un concepto modal. Es decir, la universalidad es «(...) un estadio en el camino de la investigación, el hallazgo, la formación, el desarrollo y la realización ampliada de la ley» ${ }^{30}$.

\section{LA APLICACIÓN DE LA LEY}

A partir de lo que hemos argumentado hasta aquí, puede verse que, para determinar la universalidad de la ley, es de enorme importancia la cuestión de su aplicabilidad exhaustiva. De este modo, se vinculan indisolublemente la forma de la ley, es decir, su aptitud para ser aplicable a cada caso, con la materia, o sea, el contenido que adquiere la ley en cada aplicación particular. Sin aplicabilidad no hay contenido, pero sin contenido tampoco hay aplicación y, por tanto, universalidad en sentido estricto.

COHEN rechaza de este modo la objeción de que la ley sería un mero formalismo, mostrando la relación dialéctica que existe entre forma y contenido, desde el punto

28 G. GigliotTi, 1994.

$29 \mathrm{ErW}, 272$.

$30 \ll(. .$.$) eine Stufe auf dem Wege der Forschung, der Findung und der Ausbildung und Ausdehnung und$ erweiterten Durchführung des Gesetzes» (ErW, 275). 
de vista de la universalidad ${ }^{31}$. La universalidad no puede ser pensada metodológicamente sin considerar al mismo tiempo el carácter necesario de la ley. Una característica conlleva la otra, porque la ley es aplicable a todos los casos en ella tipificados, siempre y cuando contenga en sí las indicaciones metodológicas para encontrarlos. Si la ley tipifica correctamente los casos que entran en su órbita, entonces es aplicable con necesidad lógica.

COHEN define el procedimiento de aplicación de la ley como un razonamiento silogístico, cuya necesidad se funda en la corrección misma del argumento. Se trata de un silogismo cuya premisa mayor es la ley a aplicar, la menor es el caso particular que se considera y la conclusión es la aplicación misma, con ciertos matices sobre el carácter de la premisa inicial, pero con un eco del silogismo práctico aristotélico. La necesidad es la consumación y la certificación de la universalidad misma de la ley. Por eso COHEN se niega a admitir que el Derecho tenga lagunas, que impedirían su aplicación a determinados casos. En sus propias palabras:

«La validez objetiva de esta universalidad de la norma se muestra en el principio fundamental del Derecho, de que el juez no puede decir que la ley tendría una laguna, y que le impediría encontrar el Derecho. Esta afirmación está prohibida para el juez; contradice el concepto de la norma legal, que el juez debe cumplir, administrar e interpretar. Él es juez y no legislador. La ley le ha sido dada; y la ley es universal» ${ }^{32}$.

Es decir, el trabajo del juez en la aplicación de la ley no es otro que construir el silogismo deductivo que permita identificar el caso en cuestión, como un ejemplo más que está contenido en la norma. Éste es el significado de la hermenéutica jurídica. De este modo se desarrollan las virtualidades inagotables que contempla la ley. Por esta razón, el juez no puede excusarse de la tarea hermenéutica y demostrativa a la que está obligado por su cargo, con el pretexto de que ninguna ley contempla el caso que le ha sido confiado, y que por tanto se trata de una laguna del sistema legal ${ }^{33}$. La universalidad de la ley implica una necesidad apodíctica, porque consiste en una serie de instrucciones para la elaboración de los silogismos deductivos que permiten su aplicación ${ }^{34}$.

31 «A partir de esto resulta de nuevo comprensible que nadie puede darse por satisfecho con esta determinación de la ley a partir de la metodología de la forma, porque se extrañaría el contenido (que es en realidad una expresión incorrecta) en esta forma universal; mientras que con razón, como veremos, lo que se debería extrañar es la relación con el contenido y la transición en cierto modo de la forma en el contenido. No basta con decir que el concepto de la ley exigiría la universalidad; sino que lo válido es enseñar expresamente que la universalidad tiene el sentido metodológico más preciso: el hacer deducible toda particularidad, que forma parte del problema que nos ocupa, a partir de sí misma. Éste es el sentido modal de la universalidad» («Daraus wird es aber wiederum verständlich, dass man mit dieser Bestimmung des Gesetzes aus der Methodik der Form heraus sich nicht zufrieden geben mochte, dass man in einem falschen Ausdruck des gefühlten Mangels den Inhalt in dieser allgemeinen Form vermisste; während man mit Recht, wie wir sehen wollen, den Zusammenhang mit dem Inhalt und den Übergang gleichsam der Form in den Inhalt vermissen durfte. Es genügt nicht zu sagen, dass der Begriff des Gesetzes die Allgemeinheit fordere; sondern es gilt ausdrücklich zu lehren, dass die Allgemeinheit den prägnanten methodischen Sinn hat: alle Einzelheit, die dem betreffenden Problema angehört, aus sich ableitbar zu machen. Das ist der modale Sinn der Allgemeinheit», ErW, 276).

32 «Die tatsächliche Geltung dieser Allgemeinheit der Norm zeigt sich in dem Grundsatze des Rechts, dass der Richter nicht sagen darf, das Gesetz habe eine Lücke, und er könne das Recht nicht finden. Dieser Spruch ist der Richter versagt; er widerspricht dem Begriffe der Rechtsnorm, die er zu befolgen, zu verwalten und auszulegen hat. Er ist Richter und nicht Gesetzgeber. Das Gesetz ist ihm gegeben; und das Gesetz ist allgemein» $(E r W, 277)$.

33 WINTER, 1980: 351, 382-384.

$34 \mathrm{ErW}, 278$. 


\section{EL VALOR MODAL DEL TIEMPO EN LA LEY JURÍDICA}

Otra diferencia importante entre las normas legales y las leyes naturales tiene que ver con el valor del tiempo como categoría modal. Se trata de la dimensión temporal de ambas formas de legalidad, pero de acuerdo con la peculiaridad y especificidad de su estructura lógica. El análisis de la estructura de las normas legales nos permite concluir que están referidas necesariamente al futuro, porque prescriben una serie de acciones a realizar, tanto para cumplir con ellas como para sancionar a quienes no lo hagan ${ }^{35}$. Es decir, las normas legales están orientadas a producir determinadas consecuencias en el futuro ${ }^{36}$.

Si no se hace referencia a esta dimensión temporal, no puede siquiera hablarse con sentido de normas legales. Las leyes naturales, en cambio, son intemporales, porque explican la ocurrencia de fenómenos según regularidades que deben ser válidas para todo tiempo. En esto radica la universalidad y necesidad de las leyes naturales, a diferencia de la universalidad y la necesidad apodíctica de las normas legales que se ha analizado más arriba. De este modo, hemos retornado a la objeción de ZiTELMANN, basada en la consideración de las normas legales como una forma de las leyes naturales.

Desde el punto de vista de COHEN, una concepción de este tipo implicaría la negación de la autoconciencia, porque la voluntad del Estado no sería la expresión de una voluntad pura, resultado de la deliberación y la legislación de los representantes de todos los ciudadanos, según el procedimiento establecido en la Constitución ${ }^{37}$. Pero el carácter temporal de las normas legales —o sea, su referencia interna al futuro- las diferencia claramente de las leyes naturales. Entonces, la ética también encuentra su justificación en la estructura lógica de las normas legales ${ }^{38}$. Si la ley es la tarea de la autoconciencia, entonces pierde sentido la concepción psicologista y naturalista de la autoconciencia del individuo, en tanto que sujeto de la moralidad ${ }^{39}$.

La orientación al futuro es la fuente del carácter progresista del socialismo y de la resistencia contra las tendencias políticamente reaccionarias presentes en todo Estado. Si el valor modal del futuro permite fundamentar a la autoconciencia universal, entonces también es determinante para la realización del carácter moral del Estado ${ }^{40}$. De allí toma su fuerza la virtud de la valentía, que sostiene el optimismo en la lucha histórica por el establecimiento del Estado democrático de Derecho. ${ }^{41}$ Éste es el sentido de las siguientes afirmaciones de SCHMID:

«Frente a la realidad llena de sufrimiento el hombre está llamado a alcanzar el ideal con su trabajo y a no desesperar. El optimismo es la salida de la virtud de la valentía. (...) Para el socialismo, la virtud política de la valentía significa ahora que no deben ser elegidos el

35 DREYER, 1985: 124.

$36 \mathrm{ErW}, 279$.

37 En el nivel de la fundamentación del sistema de COHEN, esto implicaría ante todo la contradicción de la ley fundamental de la verdad, que prescribe que la filosofía teórica y la filosofía práctica deben conservar sus respectivos ámbitos de trabajo y no deben de ninguna manera invadir el de otro (ErW, 83-108).

$38 \mathrm{ErW}, 281$.

39 ErW, 282. COHEN criticó duramente el psicologismo de su maestro F. A. LANGE en COHEN, 1914.

40 P. FIORATO ha explorado los fundamentos lógico-sistemáticos del mesianismo de CoHEN, en particular en relación con el problema del tiempo (FIORATO, 1993 y 1994).

${ }^{41} \operatorname{ErW}, 552-598$. 
camino de la revolución y de la mala utopía, sino más bien aquél de la reforma. La virtud de la valentía hace posible la creencia en la capacidad de reforma de la realidad llena de sufrimiento. Ella hace posible una visión optimista del mundo y de este modo le abre al hombre la esperanza en un futuro mejor, es decir, la esperanza en la realización del fin de la historia mundial, que es el Estado como el Estado de la humanidad. De este modo, la virtud de la valentía se convierte en una "virtud de la historia mundial" 》 $(E r W, 558){ }^{42}$.

\section{CONCLUSIONES}

Luego de la argumentación desarrollada a lo largo de este trabajo, puede verse que la ética de COHEN resiste a la acusación de ser un formalismo abstracto, porque su concepto de ley jurídica opera como mediador entre cada individuo particular y la universalidad de sus conciudadanos. En primer lugar, el ciudadano individual participa de la expresión de la voluntad universal del Estado, al elegir a sus representantes en el Parlamento por medio del sufragio universal. Esta integración en la tarea de la declaración de la voluntad estatal implica la realización constante de la autoconciencia, que es el sujeto universal del Estado como persona jurídica. Los ciudadanos entonces son en realidad colegisladores y regulan comunitariamente la vida del Estado.

Pero esto no significa que, una vez que se haya realizado la autoconciencia, la ley promulgada se convertiría en una abstracción vacía de contenido y desprovista de toda relevancia para la vida cotidiana de los ciudadanos de carne y hueso. Por el contrario, luego del primer momento de la producción de la ley en el Parlamento, debe seguir el momento de su aplicación por el juez a cada caso particular. De este modo, toma realidad concreta y palpable la autoconciencia, es decir, la comunidad universal que une a todos los ciudadanos con los lazos de la ley que ellos mismos han proclamado. La ley entonces es una mediación dialéctica que integra en sí a los momentos de la particularidad y la universalidad; es decir, que cumple con el objetivo fundamental que COHEN le asigna a la ética.

\section{BIBLIOGRAFÍA}

\section{Bibliografía primaria}

Cohen, H., 1907: Ethik des reinen Willens, en 2002, Herman Cohen. Werke, herausgegeben vom Hermann-Cohen-Archiv am Philosophischen Seminar der Universität Zürich unter der Leitung von Helmut Holzhey, Band 7, System der Philosophie. 1. Teil. Ethik des reinen Willens, Georg Olms Verlag, Hildesheim-Zürich-New York. Einleitung von Peter A. Schmid. Nachdruck der 2. revidierten Auflage (Berlin, 1907, Bruno Cassirer). Citado con la abreviatura $E r W$.

42 «Angesichts der leidensvollen Realität ist der Mensch dazu angerufen, das Ideal zu erarbeiten und nicht zu verzweifeln. Der Optimismus ist der Ausfluss der Tugend der Tapferkeit (...). Für den Sozialismus bedeutet die politische Tugend der Tapferkeit nun, dass nicht der Weg der Revolution und der schlechten Utopie gewählt werden soll, sondern derjenige der Reformation. Die Tugend der Tapferkeit ermöglicht den Glauben an die Reformfähigkeit der leidensvollen Realität. Sie ermöglicht eine optimistische Weltsicht und eröffnet so dem Menschen die Hoffnung auf eine bessere Zukunft, d.h., die Hoffnung auf die Realisierung des Ziels der Weltgeschichte, des Staates als Staat der Menschheit. Dadurch wird die Tugend der Tapferkeit zur, Tugend der Weltgeschichte» (ErW, 558). Cfr. SCHMID, 1993: 83-84. 
Cohen, H., (1914), Einleitung mit kritischem Nachtrag, Leipzig, Verlag von Friedrich Brandstetter.

\section{Bibliografía secundaria}

BIERLING, E. R., 1894 ss.: Juristische Prinzipienlehre, Leipzig und Freiburg i. B., J. C. B. Mohr (Paul Siebeck).

Dreyer, M., 1985: Die Idee Gottes im Werk Hermann Cohens, Königstein/Ts., Verlag Anton Hain Meisenheim GmbH.

FIORATO, P., 1993: Geschichtliche Ewigkeit. Ursprung und Zeitlichkeit in der Philosophie Hermann Cohens, Würzburg, Königshausen \& Neumann.

— 1994: «Die Gegenwart muß indessen zur Zukunft werden“. Über die "logischen” Grundlagen des Cohenschen Messianismus», in E. W. OrTH y H. Holzhey (Hrsg.), 1994, Neukantianismus. Perspektiven und Probleme, Würzburg, Königshausen \& Neumann, 366-378.

GigliotTi, G., 1994: «Ethik und das Faktum der Rechtswissenschaft bei Hermann Cohen», in H. HolzHEY (Hrsg.), Ethischer Sozialismus, Frankfurt, Suhrkamp, 166-184.

GuARIGLIA, O., 1992: «Kantismo», en: Enciclopedia Iberoamericana de Filosofía, vol. II: Concepciones de la ética, Madrid, Trotta, 3-72.

KANT, I., 1997: La metafísica de las costumbres, trad. esp. de Adela Cortina Orts y Jesús Conill Sancho, Madrid, Altaya.

KleIN, J., 1976: Die Grundlegung der Ethik in der Philosophie Hermann Cohens und Paul Natorps - eine Kritik des Neukantianismus, Göttingen, Vandenhoeck \& Ruprecht.

KöHNKE, K. Ch., 1986: Entstebung und Aufstieg des Neukantianismus. Die deutsche Unversitätsphilosophie zwischen Idealismus und Positivismus, Frankfurt am Main, Suhrkamp.

Lembeck, K.-H., 1994: Platon in Marburg. Platonrezeption und Philosophiegeschichtsphilosophie bei Cohen und Natorp, Würzburg, Königshausen \& Neumann.

LISSER, K., 1922: Der Begriff des Rechts bei Kant. Mit einem Anbang über Coben und Görland, Berlin, Verlag von Reuter \& Reichard.

MüLlER, C., 1994: Die Rechtsphilosophie des Marburguer Neukantianismus. Naturrecht und Rechtpositivismus in der Auseinandersetzung zwischen Hermann Cohen, Rudolf Stammler und Paul Natorp, Tübingen, J. C. B. Mohr (Paul Siebeck).

SCHMID, P., 1993: Ethik als Hermeneutik. Systematische Untersuchungen zu Hermann Cohens Rechts und Tugendlebre, Würzburg, Könighausen \& Neumann.

SIEG, U., 1994: Aufstieg und Niedergang des Marburger Neukantianismus. Die Geschichte einer philosophischen Schulgemeinschaft, Würzburg, Könighausen \& Neumann.

Willey, Th., 1978: Back to Kant. The Revival of Kantianism in German Social and Historical Thought, 1860-1914, Detroit, Wayne State University Press.

WINTER, E., 1980: Ethik und Rechtswissenschaft. Eine historisch-systematische Untersuchung zur Ethik-Konzeption des Marburger Neukantismus im Werke Hermann Cohens, Berlin, Duncker \& Humblot.

Zitelmann, E., 1879: Irrtum und Rechtsgeschäft, Leipzig, Duncker und Humblot. 